PROCEEDINGS OF THE

AMERICAN MATHEMATICAL SOCIETY

Volume 126, Number 2, February 1998, Pages 447-452

S 0002-9939(98)04076-3

\title{
ON THE DILATATION OF UNIVALENT PLANAR HARMONIC MAPPINGS
}

\author{
ALLEN WEITSMAN
}

(Communicated by Albert Baernstein II)

\begin{abstract}
It is shown that if $f$ is a univalent harmonic mapping of the unit disk onto a domain having a smooth boundary arc which is convex with respect to the domain, and if the dilatation has modulus 1 on the arc, then the arc must be a line segment.
\end{abstract}

\section{INTRODUCTION}

In 1952, E. Heinz [H] used planar univalent harmonic mappings in the study of the Gaussian curvature of nonparametric minimal surfaces over the unit disk. Since that time, the study of univalent harmonic mappings has gained much attention in its own right.

Let $\mathcal{U}$ be the unit disk $\{z:|z|<1\}$ and $f=u+i v$ be a univalent (orientation preserving) harmonic mapping of $\mathcal{U}$ onto a bounded domain $\Omega$. Then $f=h+\bar{g}$ where $h$ and $g$ are analytic in $\mathcal{U}$. Furthermore, $f$ satisfies the equation

$$
\overline{f_{\bar{z}}}=a f_{z}
$$

in $\mathcal{U}$, where $a(z)=g^{\prime}(z) / h^{\prime}(z)$, and $|a(z)|<1$ in $\mathcal{U}$. We shall assume henceforth that the univalent harmonic mappings are orientation preserving.

By Fatou's theorem, $f$ and $a$ must have radial limits a.e. on $\partial \mathcal{U}$. However, even if $\partial \Omega$ is smooth, the boundary correspondence between $\partial \mathcal{U}$ and $\partial \Omega$ can be quite pathological. In particular the radial limit function $\hat{f}\left(e^{i \theta}\right)$ can have discontinuities, and thus $f$ need not extend to a homeomorphism of the closures of $\mathcal{U}$ and $\Omega$. A general description of the boundary behavior of univalent harmonic mappings of $\mathcal{U}$ onto regions having locally connected boundary is given in [HS2, Theorem 4.3], [HS1, Lemma 3.1]. If $\partial \Omega$ is locally connected, then outside a countable set $E$ of the boundary, $f$ has unrestricted limits through points of $\mathcal{U}$. For points in $E$, the limits from the left and right (avoiding other points in $E$ ) for $\hat{f}$ exist and are different; the cluster sets at points of $E$ are line segments joining these left and right limits. At points of $\partial \mathcal{U} \backslash E$, the one sided limits (again taken outside of the set $E$ ) of $\hat{f}$ are equal.

In [HS1] Hengartner and Schober studied the boundary behavior of harmonic mappings and proved that if $\Omega$ is convex and $a(z)$ is a finite Blaschke product, then

Received by the editors November 18, 1995 and, in revised form, May 6, 1996 and August 6, 1996.

1991 Mathematics Subject Classification. Primary 30C62, 31A05, 31A20, 49Q05.

Key words and phrases. Harmonic mappings, dilatation, minimal surfaces.

(c)1998 American Mathematical Society 
$\Omega$ must be a polygon [HS1, Theorem 3.3]. In the present paper we shall localize the argument of Hengartner and Schober to study the behavior of $f$ if $\partial \Omega$ contains a $C^{1}$ arc $\gamma$ which is convex with respect to $\Omega$. By this we mean that any line joining distinct points of $\gamma$ lies, with the exception of its endpoints, in $\Omega$, and that the line $L$ joining the endpoints of $\gamma$ separates $\Omega$ into two domains whose boundaries share only $L$ in common.

Theorem 1.1. Let $f$ be a univalent harmonic mapping of $\mathcal{U}$ onto a domain $\Omega$, $a(z)$ as in (1.1), and $\gamma \subset \partial \Omega$ a $C^{1}$ arc, which is convex with respect to $\Omega$. Suppose that for any point $\zeta$ on $\gamma$, and any sequence $\zeta_{n} \rightarrow \zeta$ in $\Omega$, if $\zeta_{n}=f\left(z_{n}\right)$, we have $\left|a\left(z_{n}\right)\right| \rightarrow 1$. Then $\gamma$ must be a line segment.

It should be pointed out that in Theorem 1.1, $a(z)$ could have radial limits of modulus 1 almost everywhere without $\gamma$ being a line segment. Indeed, if $\Phi(\theta)$ is a sense preserving homeomorphism of $[\theta, 2 \pi)$ onto $[\theta, 2 \pi)$ such that $\Phi^{\prime}(\theta)=0$ a.e., then by a theorem of Laugesen [L, Theorem 1], the Poisson integral $f$ of $\Phi$ gives a univalent harmonic mapping of $\mathcal{U}$ onto itself, such that $\left|a\left(r e^{i \theta}\right)\right| \rightarrow 1$ as $r \rightarrow 1^{-}$, for a.e. $\theta$.

From the standpoint of minimal surfaces, Theorem 1.1 has an interesting consequence. Let $S$ be a nonparametric minimal surface over a simply connected domain $\Omega$ given by

$$
S=\{(u, v, F(u, v)): u+i v \in \Omega\},
$$

where we have identified $R^{2}$ with the complex plane in describing the domain $\Omega$ of $F$. Then, in $\Omega, w=F(u, v)$ satisfies the minimal surface equation

$$
\left(1+\left(\frac{\partial F}{\partial v}\right)^{2}\right) \frac{\partial^{2} F}{\partial u^{2}}-2 \frac{\partial F}{\partial u} \frac{\partial F}{\partial v} \frac{\partial^{2} F}{\partial u \partial v}+\left(1+\left(\frac{\partial F}{\partial u}\right)^{2}\right) \frac{\partial^{2} F}{\partial v^{2}} .
$$

By the Weierstrass representation, we may reparametrize $S$ in parametric form by a pair $(\omega, G)$, where $G$ is meromorphic in $\mathcal{U}$ and $\omega$ is analytic in $\mathcal{U}$, having its zeros at the poles of $G$ with twice the multiplicity of the poles. The coordinate functions are then given by

$$
\begin{gathered}
u(z)=\operatorname{Re} \frac{1}{2} \int^{z} \omega(\zeta)\left(1-G(\zeta)^{2}\right) d \zeta \\
v(z)=\operatorname{Re} \frac{i}{2} \int^{z} \omega(\zeta)\left(1+G(\zeta)^{2}\right) d \zeta \\
w(z)=\operatorname{Re} \int^{z} \omega(\zeta) G(\zeta) d \zeta
\end{gathered}
$$

The functions $u, v, w$ are harmonic in $\mathcal{U}$, and with $S$ being a graph, the first two coordinate functions determine a univalent harmonic map. Furthermore, the stereographic projection of the Gauss map $\mathcal{G}(u, v)$ of the surface pulls back by the relation

$$
G(z)=\mathcal{G}(u(z), v(z))=i / \sqrt{a(z)}
$$

where $G$ is as above in the Weierstass representation, and $a$ is as in (1.1). Thus, by (1.3), in order for a univalent harmonic mapping $f$ to arise in this way from a minimal surface, $a(z)$ must be the square of an analytic function. 
From Theorem 1.1 we obtain the following

Corollary 1.1. Let $S$ be a nonparametric minimal surface over a simply connected domain $\Omega$ with $\gamma \subset \partial \Omega, \gamma$ a $C^{1}$ convex curve. If the Gauss map satisfies $|\mathcal{G}(u, v)| \rightarrow$ 1 as $u+i v \rightarrow \gamma$, then $\gamma$ is a line segment.

In other words, if the normals to the surface tend to the horizontal over $\gamma$, then $\gamma$ is a line segment. The prototype for this situation is Scherk's surface which has the shape of a saddle over a square with $|\mathcal{G}| \rightarrow 1$ at the boundary.

Corollary 1.1 is somewhat reminiscent of a result of Finn $[\mathrm{F}]$, which says that if the height function $F$ in (1.2) has the property that $F(u, v) \rightarrow \infty$ as $u+i v$ tends to an arc $\gamma$ of the boundary of $\Omega$, then $\gamma$ must be a line segment. On the other hand by tilting Scherk's surface, we obtain nontrivial smooth concave $\operatorname{arcs} \gamma$ such that $|\mathcal{G}(u, v)| \rightarrow 1$ at all points of $\gamma$.

\section{Proof of Theorem 1.1}

We assume that $\gamma$ is not a line segment. We may assume further that $\Omega$ is the domain whose boundary consists of $\gamma$ and the line segment $L$ joining the endpoints of $\gamma$. Indeed, if we denote this domain by $\Omega_{0}$ and let $\varphi$ be a 1-1 conformal mapping of $\mathcal{U}$ onto $f^{-1}\left(\Omega_{0}\right)$, then $f(\varphi(z))$ is again a univalent harmonic mapping of $\mathcal{U}$ onto $\Omega_{0}$ having $\gamma$ as a boundary arc. Replacing $f$ by $f \circ \varphi$ (which for simplicity we again denote by $f=h+g$ ), then the new $a(z)$ defined by (1.1) again satisfies $|a(z)| \rightarrow 1$ when $f(z)$ tends to points of $\gamma$.

Now, as shown by Abu-Muhanna and Lyzzaik [AL, Theorem 1], $\int_{\sigma}\left|h^{\prime}\right||d z|<\infty$, $\int_{\sigma}\left|g^{\prime}\right||d z|<\infty$ for almost all radii $\sigma$ from 0 to $\partial \mathcal{U}$. Also, by the aforementioned theorem of Hengartner and Schober, $f$ has unrestricted limits at each point of $\partial \mathcal{U}$ except at countably many points, at which the cluster sets are line segments. Thus, we may take endpoints $w_{1}, w_{2}$ of a closed proper subarc $\gamma_{0}$ of $\gamma$, where $w_{1}$ and $w_{2}$ are arbitrarily near the two endpoints of $\gamma$ and such that there are distinct radii $\sigma_{1}, \sigma_{2}$ from 0 to $\partial \mathcal{U}$ such that $f(z) \rightarrow w_{j}$ along $\sigma_{j}, j=1,2$, and

$$
\int_{\sigma_{j}}\left|h^{\prime}(z)\right||d z|<\infty, \quad \int_{\sigma_{j}}\left|g^{\prime}(z)\right||d z|<\infty, \quad j=1,2 .
$$

Let $S$ denote the sector in $\mathcal{U}$ bounded by $\sigma_{1}, \sigma_{2}$, and $\Gamma=\left\{e^{i t}: \alpha \leq t \leq \beta\right\}$, where $\Gamma$ is the arc for which the radial limits of $f$ are in $\gamma_{0}$.

Let $0<\rho<1$ such that $|a(z)|=\rho$ for some $z \in S$, and choose $\rho$ so that $a^{\prime}(z) \neq 0$ when $|a(z)|=\rho$. Let $S_{\rho}$ be the subset of $S$ where $|a(z)|>\rho$, and $\delta=\sup _{z \in S_{\rho}} \operatorname{dist}(z, \Gamma)$. Choose $\rho$ large enough so that if $z_{1}, \ldots, z_{n}$ are the zeros of $a(z)$ in $S$, then $\operatorname{dist}\left(z_{j}, \Gamma\right)>\delta, j=1, \ldots, n$. Let $T_{\rho}$ be the component of $S_{\rho}$ whose boundary contains $\Gamma$. Such a component must exist, since $|a(z)|=1$ on $\Gamma$. Furthermore, $\partial T_{\rho}$ contains a level arc $C_{\rho}$ which extends from a point $z_{1} \in \sigma_{1}$ to a point $z_{2} \in \sigma_{2}$. Let $\sigma_{1}(\rho)$ be the ray from 0 to $z_{1}$ and $\sigma_{2}(\rho)$ be the ray from $z_{2}$ to 0 . Let $\Sigma_{\rho}$ be the contour obtained by joining $\sigma_{1}(\rho), C_{\rho}$, and $\sigma_{2}(\rho)$.

We may parameterize $C_{\rho}$ by $z_{\rho}(t)=r(t) e^{i t}, \alpha_{\rho} \leq t \leq \beta_{\rho}$ for $\rho$ sufficiently close to 1 . Indeed, it follows from the Hopf maximum principle that $\partial|a| / \partial r$ is strictly positive near $\Gamma$, so $C_{\rho}$ is strictly starlike with respect to 0 for $\rho$ close to 1 . Let $M$ denote a measurable subset of the plane. Then for each $\rho$ close to 1 , we define 
measures $\mu_{\rho}$ having respective supports on $\Sigma_{\rho}$ by

$$
\mu_{\rho}(M)=\left(1-\rho^{2}\right) \int_{M \cap \Sigma_{\rho}} f_{z} d z
$$

Then, on $C_{\rho}$,

$$
\left(1-\rho^{2}\right) f_{z} d z=\left(1-|a|^{2}\right) f_{z} d z=d f-\overline{a d f},
$$

so $\mu_{\rho}=\mu_{\rho}^{(1)}+\mu_{\rho}^{(2)}$, where $\mu_{\rho}^{(1)}$ has support on $\sigma_{1}(\rho) \cup \sigma_{2}(\rho), \mu_{\rho}^{(2)}$ has support on $C_{\rho}$, and

$$
\begin{aligned}
& \mu_{\rho}^{(1)}(F)=\left(1-\rho^{2}\right) \int_{F} f_{z} d z, \quad F \subseteq \sigma_{1}(\rho) \cup \sigma_{2}(\rho), \\
& \mu_{\rho}^{(2)}(F)=\int_{F} d f-\overline{a d f}, \quad F \subseteq C_{\rho} .
\end{aligned}
$$

Since $f_{z}$ is analytic,

$$
\int_{\Sigma_{\rho}} z^{k} d \mu_{\rho}=0, \quad k=0,1,2, \ldots
$$

By (2.1) and (2.2), it follows that

$$
\mu_{\rho}^{(1)} \rightarrow 0 \quad \text { as } \rho \rightarrow 1^{-} .
$$

On $C_{\rho}$

$$
\left|d \mu_{\rho}\right|=\left|d \mu_{\rho}^{(2)}\right|=\left(1-|a|^{2}\right)\left|f_{z}\right|\left|d z_{\rho}\right| .
$$

Since $a(z)$ is analytic across $C_{\rho}$, we have

$$
\int_{C_{\rho}}\left|d z_{\rho}\right|=O(1) \quad(\rho \rightarrow 1) .
$$

Also,

$$
1-|a(z)|^{2} \leq O\left(1-|z|^{2}\right)
$$

uniformly as $z$ tends to points of $\Gamma$.

By Poisson's formula, for $z$ near $\Gamma$,

$$
\left|f_{z}(z)\right|=O(1-|z|) \text {. }
$$

Thus, by $(2.6)-(2.9)$, for a sequence $\rho_{n} \rightarrow 1^{-}$, the sequence $\mu_{\rho_{n}}^{(2)}$ converges weakly to a measure $\mu$ supported on $\Gamma$. Taking (2.5) into account, we then have that $\mu_{\rho_{n}}=\mu_{\rho_{n}}^{(1)}+\mu_{\rho_{n}}^{(2)} \rightarrow \mu$ weakly. Since $\mu$ has support on $\Gamma,(2.4)$ implies that

$$
\int_{\partial \mathcal{U}} z^{k} d \mu=0, \quad k=0,1,2, \ldots
$$

By the F. and M. Riesz theorem, $\mu$ is an absolutely continuous measure.

From (2.3) it follows that if $\hat{f}$ denotes the radial limit function for $f$, then for some constant $C$,

$$
\int_{\alpha}^{t} d \mu\left(e^{i t}\right)=\hat{f}\left(e^{i t}\right)-\overline{a\left(e^{i t}\right) \hat{f}\left(e^{i t}\right)}+\int_{\alpha}^{t} \overline{\hat{f}\left(e^{i \tau}\right) d a\left(e^{i \tau}\right)}+C
$$

a.e. on $[\alpha, \beta]$. Let $\psi(t)=\int_{\alpha}^{t} d \mu\left(e^{i t}\right)$. 
Since $\mu$ is absolutely continuous, and the integral on the right of (2.11) is an absolutely continuous function, it follows that there exists an absolutely continuous function $\varphi(t)$ such that

$$
\psi(t)=\varphi\left(e^{i t}\right)+\int_{\alpha}^{t} \overline{\hat{f}\left(e^{i \tau}\right) d a\left(e^{i \tau}\right)}+C \quad(\alpha \leq t \leq \beta),
$$

where

$$
\varphi(t)=\hat{f}\left(e^{i t}\right)-\overline{a\left(e^{i t}\right) \hat{f}\left(e^{i \tau}\right)} \quad \text { a.e. on }[\alpha, \beta] .
$$

Now, (2.10) implies that if $F(z)=\int_{0}^{2 \pi} \frac{d \mu\left(e^{i t}\right)}{1-z e^{-i t}}$, then $F$ is analytic and is also the Poisson integral of $\mu$;

$$
F\left(r e^{i \theta}\right)=\int_{0}^{2 \pi} P_{r}(\theta-t) d \mu\left(e^{i t}\right)
$$

which is 0 on an $\operatorname{arc}$ of $\partial \mathcal{U}$. Thus,

$$
\mu \equiv 0
$$

Following [HS1, p.201], we take a branch of $\sqrt{a}$ on $\Gamma$, and note that for a.e. $t \in[\alpha, \beta]$,

$$
\sqrt{a\left(e^{i t}\right)} \varphi(t)=\sqrt{a\left(e^{i t}\right)}\left(\hat{f}\left(e^{i t}\right)-\overline{a\left(e^{i t}\right) \hat{f}\left(e^{i t}\right)}=2 i \operatorname{Im}\left(a\left(e^{i t}\right) \hat{f}\left(e^{i t}\right)\right) .\right.
$$

As mentioned in $\S 1$, on a set $E$ in $\partial \mathcal{U}$, which excludes perhaps a countable set, $f$ has unrestricted limits $(=\hat{f})$. Let $E_{0} \subset E$ be those points in $\Gamma$. We may fix a branch of $\arg \sqrt{a}$ and $\arg \hat{f}$, the latter being inherited from a branch of the argument on $\gamma_{0}$. By (2.13), it follows that at any point $e^{i t} \in E_{0}$,

$$
\frac{1}{2} \arg a\left(e^{i t}\right)+\arg \left(\hat{f}\left(e^{i t^{+}}\right)-\hat{f}\left(e^{i t^{-}}\right)\right)=k \pi
$$

for some integer $k$. Since $a$ is analytic in a full neighborhood of $\Gamma$, the variation of its argument is finite, and since $|a|=1$ on $\Gamma$ and $|a|<1$ in $\mathcal{U}$, it follows from the Cauchy-Riemann equations that $\arg a\left(e^{i t}\right)$ is increasing for $t \in[\alpha, \beta]$. Also, if

$$
\alpha(t)=\inf _{\tau \geq t, e^{i \tau} \in E_{0}} \arg \left(\hat{f}\left(e^{i \tau^{+}}\right)-\hat{f}\left(e^{i \tau^{-}}\right)\right),
$$

then, since $\gamma_{0}$ is convex and $C^{1}, \alpha(t)$ is nondecreasing and bounded for $t \in[\alpha, \beta]$. Thus (2.14) can hold for only finitely many $k$, and we deduce that $E_{0}$ is a finite set.

Following [HS1, p.204], we next deduce that $\hat{f}$ is constant on $\Gamma$. We observe from (2.12) that on $\Gamma \backslash E_{0}$,

$$
0=\sqrt{a\left(e^{i t}\right)} \psi^{\prime}(t)=\sqrt{a\left(e^{i t}\right)} \varphi^{\prime}(t)+\sqrt{a\left(e^{i t}\right)} \overline{\hat{f}\left(e^{i t}\right) a^{\prime}\left(e^{i t}\right)} .
$$


Suppose that $e^{i t} \notin E_{0}$. Then from (2.15), and the fact that $E_{0}$ is a finite set,

$$
\begin{aligned}
0= & \lim _{h \rightarrow 0}\left(\sqrt{a\left(e^{i t}\right)} \frac{\varphi(t+h)-\varphi(t)}{h}\right)+\sqrt{a\left(e^{i t}\right)} \hat{\hat{f}\left(e^{i t}\right) a\left(e^{i t}\right)} \\
= & \lim _{h \rightarrow 0}\left(\sqrt{a\left(e^{i t}\right)} \frac{\hat{f}(t+h)-\hat{f}(t)}{h}-\sqrt{a\left(e^{i t}\right)} \frac{\hat{f}\left(e^{i(t+h)}\right)-\hat{f}\left(e^{i t}\right)}{h}\right) \\
& -\sqrt{a\left(e^{i t}\right)} \overline{a^{\prime}\left(e^{i t}\right) \hat{f}\left(e^{i t}\right)}+\sqrt{a\left(e^{i t}\right)} \overline{\hat{f}\left(e^{i t}\right) a^{\prime}\left(e^{i t}\right)} \\
= & 2 i \lim _{h \rightarrow 0} \operatorname{Im}\left(\sqrt{a\left(e^{i t}\right)} \frac{\hat{f}\left(e^{i(t+h)}\right)-\hat{f}\left(e^{i t}\right)}{h}\right) .
\end{aligned}
$$

If there is an arc $A=\left\{e^{i t}: \alpha_{1} \leq t \leq \beta_{1}\right\} \subseteq \Gamma \backslash E_{0}$ such that $\hat{f}\left(e^{i \alpha_{1}}\right) \neq \hat{f}\left(e^{i \beta_{1}}\right)$, there exists a sequence $t_{k} \in\left(\alpha_{1}, \beta_{1}\right)$ such that $d \hat{f}\left(e^{i t_{k}}\right) / d t$ does not exist or is not 0. Then,

$$
\alpha_{k}=\lim _{n \rightarrow \infty} \arg \frac{\hat{f}\left(e^{i\left(t_{k}+h_{k_{n}}\right)}\right)-\hat{f}\left(e^{i t_{k}}\right)}{h_{k_{n}}}
$$

exists for some $h_{k_{n}} \rightarrow 0$. By (2.16), $\alpha_{k}$ must be an integer multiple of $\pi$.

Thus, if $\tilde{\alpha}(t)=\inf _{t \leq t_{k}} \alpha_{k}$, then, as before, $\arg \sqrt{a\left(e^{i t}\right)}$ is increasing and $\tilde{\alpha}(t)$ is nondecreasing. Since $\tilde{\alpha}(t)+\arg \sqrt{a\left(e^{i t}\right)}$ is a multiple of $\pi$, it follows that $A$ has finitely many points. Since $\hat{f}$ is continuous on $A$, it is constant there. Finally, since the cluster set of $\hat{f}$ at each point of $E_{0}$ is a line segment, and $\gamma$ is $C^{1}$, we find that $\gamma$ must be a line segment, a contradiction.

\section{REFERENCES}

[AL] Y. Abu-Muhanna and A. Lyzzaik, The boundary behaviour of univalent harmonic maps, Pac. Jour. 141 (1990), 1-20. MR 90m:30044

[F] R. Finn, New estimates for equations of minimal surface type, Arch. Rat. Mech. Anal. 14 (1963), 337-375. MR 28:336

$[\mathrm{H}] \quad$ E. Heinz, Über die Lösungen der Minimalflächengleichung, Nachr. Akad. Wiss. Göttingen Math. Phys. Kl. II (1952), 51-56. MR 14:885c

[HS1] W. Hengartner and G. Schober, On the boundary behavior of orientation preserving harmonic mappings, Complex Variables 5 (1986), 197-208. MR 88a:30067

[HS2] _ Harmonic mappings with given dilation, J. Lond. Math. Soc. 33 (1986), 473-483. MR 87j:30037

[L] R. Laugesen, Planar harmonic maps with inner and Blaschke dilatations, to appear.

Department of Mathematics, Purdue University, West Lafayette, Indiana 47907

E-mail address: weits@math.purdue.edu 